Pharyngeal swabs from a subset of participants were collected and assessed for commensal Neisseria prevalence, as well as characterisation of antimicrobial susceptibility to ciprofloxacin, cefixime, ceftriaxone, and cefpodoxime.

Results Thirty-nine MSM were surveyed. Median age was 23, $72.7 \%$ have sex only with men, and $18.2 \%$ have exchanged sex to receive food, money, or work. Any antibiotic use was $82 \%$ in the past 6 months and $33 \%$ in the past 30 days. In the past 6 months, use of cefixime and ceftriaxone was $15 \%$ and $9 \%$, respectively. Ever use of antibiotics without a prescription was $64 \%$.At least one commensal Neisseria was isolated from every participant, with prevalence of $N$. cinerea (14.3\%), N. flavescens $(57.1 \%)$, N. mucosa (28.6\%), N. subflava (50\%). Using CLSI MIC breakpoints for N. gonorrhoea as a proxy, "reduced susceptibility" to ciprofloxacin, cefixime, ceftriaxone, and cefpodoxime was found among $47.1 \%$, $11.8 \%, 11.8 \%$, and $29.4 \%$ of all isolates, respectively; $52.9 \%$ were "resistant" to ciprofloxacin.

Conclusion MSM of Hanoi were likely to take antibiotics without a prescription. Commensal Neisseria were very prevalent from pharyngeal samples; some may have reduced susceptibility or resistance to common antibiotics. More data is needed to appropriately interpret antimicrobial susceptibility for these commensals.

\section{P1.24 MYCOPLASMA GENITALIUM AND TRICHOMONAS VAGINALIS DETECTION IN A COHORT OF MEN WHO HAVE SEX WITH MEN IN BELGIUM: EVALUATION OF THE DIAGENODE S-DIAMGTV MULTIPLEX KIT}

Irith De Baetselier, Hilde Smet, Bea Vuylsteke, Tania Crucitti. Institute of Tropical Medicine, Antwerp, Belgium

\subsection{6/sextrans-2017-053264.132}

Introduction: Mycoplasma genitalium (MG) and Trichomonas vaginalis (TV) are Sexually Transmitted Infections (STIs) frequently detected in Non-chlamydial Non-gonococcal urethritis, however the prevalence of MG and TV in urine (U), anorectal (A) and pharyngeal (P) samples is unknown among men who have sex with men (MSM) in Belgium. The objective was to evaluate the MG/TV multiplex kit of Diagenode on the three different sample types.

Methods In the context of an ongoing study among MSM, UA-P samples are collected every three months to test for STIs including MG and TV. DNA extraction is performed on the Abbott m2000sp system. The DNA extracts are tested in both an accredited in-house MG pdhD- gene real-time PCR (RTPCR) for MG (target: pdhD gene) and the S-DiaMGTV multiplex kit of Diagenode for MG (target: mgpa and mg219 gene) and TV (target: TV-spec repeat gene). In addition, in case of a positive TV result, the sample is tested by an inhouse TV RT-PCR (TV-spec repeat gene). All amplifications were performed using the Rotor-GeneQ MDx HRM (Qiagen) platform.

Results To date, of the 1779 samples that were tested for MG/TV, 1768 provided a valid result. Only one anorectal sample was positive for TV but this result could not be confirmed by the in-house TV RT-PCR assay. MG was detected by both assays in 64 samples. The S-DiaMGTV assay missed $3 / 2 / 1$ U-A-P infections and identified additional 2/2/2 U-A-P infections. Both assays were 99.3\% concordant for MG. Up to now an overall prevalence of $4.0 \%$ for $\mathrm{MG}$ is found: $4.8 \%(29 / 598) ; 6.4 \%(37 / 578)$ and $0.7 \%$ (4/592) for U-A-P, respectively. Compared to the in-house RT-PCR the SDiaMGTV showed an overall sensitivity/specificity of 91.4\%/ 99.7\%: 89.7\% (CI 95\%: 72.7\%-97.8\%)/99.7\% (CI 95\%: 98.7\%-100\%) for urine; 94.6\% (CI 95\%: 81.8\%-99.3\%)/ 99.6\% (CI 95\%: 98.7\%-100.0\%) for anorectal and $75.0 \%$ (CI 95\%: 19.4\%-99.4\%)/99.7\% (CI 95\%: 98.8\%-100.0\%) for pharyngeal samples.

Conclusion MG but not TV is prevalent in MSM in urine and anorectal samples. The S-DiaMGTV multiplex kit is an appropriate assay to detect MG in urine and anal samples.

\section{P1.25 COLLI-PEE: A NEW DEVICE TO COLLECT FIRST-VOID URINE AT HOME FOR MOLECULAR DETECTION OF STIS. EVALUATION \& ACCEPTABILITY BY MSM IN A PREP STUDY IN BELGIUM}

${ }^{1}$ Irith De Baetselier, ${ }^{2}$ Vanessa Vankerckhoven, ${ }^{2}$ Manon De Koeijer, ${ }^{1}$ Thijs Reyniers, ${ }^{1}$ Hilde Smet, ${ }^{1}$ Bea Vuylsteke, ${ }^{1}$ Tania Crucitti. 'Institute of Tropical Medicine, Antwerp, Belgium; ${ }^{2}$ Novosanis, Wijnegem, Belgium

\subsection{6/sextrans-2017-053264.133}

Introduction The CE-labelled Colli-Pee device efficiently captures first-void urine without interruption of the urine stream and is suited for postal delivery. We aimed to evaluate the use of Colli-Pee collected urine mailed by regular post for sexually transmitted infection (STI) detection.

Methods A Belgian study cohort of men who have sex with men were asked to collect first-void urine using the Colli-Pee at home the day after the study visit. At the visit, a first-void urine sample was collected in a regular recipient to detect Neisseria gonorrhoeae (NG), Chlamydia trachomatis (CT), Mycoplasma genitalium (MG) and Trichomonas vaginalis (TV) using commercial and in house Nucleic Acid Amplification Techniques (NAATs). The Colli-Pee collector tube was sent back to the laboratory by regular post and the same NAATs were performed. A survey was completed by the participants on the usability and willingness to use the Colli-Pee device.

Results To date, 265 results were taken into account. Using Colli-Pee, one additional CT was detected and one CT was missed $(5 / 5)$ compared to the regular recipient. Two additional NG (7/5) and 2 additional MG were detected but one MG was missed using the Colli-Pee (16/15). TV was not detected in any of the urine samples. The Kappa agreement was $79,6 \%, 83 \%$ and $89,7 \%$ for CT, NG and MG, respectively. On average, Colli-Pee samples were taken 1.7 days (0-22 days) after the regular samples and were on route for an average of 4,3 days (0-17 days). $89 \%$ found the Colli-Pee easy to use, but $16 \%$ remarked that the Colli-Pee is not recyclable.

Conclusion The two STIs (CT/MG) that were missed using the Colli-Pee were only weak positive in the regular sample. More STIs were detected in the Colli-Pee samples: $1 \mathrm{CT}$, $2 \mathrm{NG}$ and $2 \mathrm{MG}$. However, the Colli-Pee samples were collected $1,2,3,8$, and unknown days later than the regular urines, we cannot exclude that participants became positive or infected during that time frame. In conclusion, we showed that the Colli-Pee can be used for home collection of firstvoid urine and postal delivery for STI testing. 